For Publisher's use

\title{
QED $\otimes$ QCD EXPONENTIATION: SHOWER/ME MATCHING AND IR-IMPROVED DGLAP THEORY AT THE LHC
}

\author{
B.F.L. WARD AND S.A. YOST \\ Department of Physics, Baylor University, Waco, TX, USA
}

\begin{abstract}
We discuss the elements of $\mathrm{QED} \otimes \mathrm{QCD}$ exponentiation and its interplay with shower/ME matching and IR-improved DGLAP theory in precision LHC physics scenarios. Applications to single heavy gauge boson production at hadron colliders are illustrated.
\end{abstract}

BU-HEPP-06-07, Oct., 2006, presented by B.F.L. Ward at ICHEP06

Given the approaching turn-on of the LHC, the issue of precision predictions for the effects of multiple gluon and multiple photon radiative processes is a more immediate. A problem of some interest is the construction of an event-by-event precision theory of potential luminosity processes such as single heavy gauge boson production processes. Presuming the LHC luminosity experimental error to reach $2 \%$ [1], the attendant theoretical precision tag on processes such as single $\mathrm{W}, \mathrm{Z}$ production should be $\frac{2}{3}-1 \%$ so that the theoretical error does not adversely affect the respective luminosity determination and the attendant precision LHC physics ${ }^{a}$. Thus, we have developed [3] a theory of the simultaneous resummation of multiple gluon and multiple photon radiative effects, $Q E D \otimes Q C D$ exponentiation, to realize systematically the needed higher order corrections on an eventby-event basis, in the presence of parton showers, to the desired accuracy.

We note that the new $Q E D \otimes Q C D$ exponentiation theory is an exact resummation theory in the spirit of the original YFS exponentiation for $Q E D$ and is an extension of the $Q C D$ exponentiation theory presented in Refs. [4]. One should compare the latter to the formal proof of exponentiation in non-Abelian gauge theories in the eikonal ap-

${ }^{a}$ We note for reference that the current precision tag for the luminosity at FNAL is at the $\sim 7 \%$ level [2]. proximation as given in Ref. [5]: the results in Ref. [4] are in contrast exact but have an exponent that only contains the leading contribution of the exponent in Ref. [5]- if desired, all of the contributions of the latter exponent can be incorporated into the exponent Ref. [4] while maintaining exactness. Our precision requirement for the LHC luminosity processes requires exact $\mathcal{O}\left(\alpha_{s}^{2}, \alpha_{s} \alpha, \alpha^{2}\right)$ $Q E D \otimes Q C D$ exponentiation in the presence of parton showers, on an event-by-event basis, realized by MC methods for realistic multiple gluon and multiple photon radiative effects. Development of such MC's is in progress.

We note that in Ref. [3] we have shown that for a process such as $q+\bar{q}^{\prime} \rightarrow V+n(G)+$ $n^{\prime}(\gamma)+X \rightarrow \bar{\ell} \ell^{\prime}+n(g)+n^{\prime}(\gamma)+X$ we have the result

$$
\begin{array}{r}
d \hat{\sigma}_{\exp }=e^{\mathrm{SUM}_{\mathrm{IR}}(\mathrm{QCED})} \\
\sum_{n, m=0}^{\infty} \int \prod_{j_{1}=1}^{n} \frac{d^{3} k_{j_{1}}}{k_{j_{1}}} \prod_{j_{2}=1}^{m} \frac{d^{3} k_{j_{2}}^{\prime}}{k^{\prime} j_{2}} \int \frac{d^{4} y}{(2 \pi)^{4}} \\
e^{i y \cdot\left(p_{1}+q_{1}-p_{2}-q_{2}-\sum k_{j_{1}}-\sum k_{j_{2}}\right)+D_{\mathrm{QCED}}} \\
\tilde{\bar{\beta}}_{n, m}\left(k_{1}, \ldots, k_{n} ; k_{1}^{\prime}, \ldots, k_{m}^{\prime}\right) \frac{d^{3} p_{2}}{p_{2}^{0}} \frac{d^{3} q_{2}}{q_{2}^{0}}
\end{array}
$$

where the new YFS $[6,7]$ residuals, defined in Ref. [3], $\tilde{\bar{\beta}}_{n, m}\left(k_{1}, \ldots, k_{n} ; k_{1}^{\prime}, \ldots, k_{m}^{\prime}\right)$, with $n$ hard gluons and $m$ hard photons, represent the successive application of the YFS expansion first for QCD and subsequently for QED. The functions $\operatorname{SUM}_{\mathrm{IR}}(\mathrm{QCED}), D_{\mathrm{QCED}}$ 
$\underset{\tilde{\beta}}{\operatorname{are}}$ given in Ref. [3]. The residuals $\tilde{\bar{\beta}}_{n, m}\left(k_{1}, \ldots, k_{n} ; k_{1}^{\prime}, \ldots, k_{m}^{\prime}\right)$ are free of all infrared singularities and the result in (1) is a representation that is exact and that can therefore be used to make contact with parton shower MC's without double counting or the unnecessary averaging of effects such as the gluon azimuthal angular distribution relative to its parent's momentum direction.

For our prototypical processes, we have the standard formula

$$
\begin{array}{r}
d \sigma_{\exp }\left(p p \rightarrow V+X \rightarrow \bar{\ell} \ell^{\prime}+X^{\prime}\right)= \\
\sum_{i, j} \int d x_{i} d x_{j} F_{i}\left(x_{i}\right) F_{j}\left(x_{j}\right) d \hat{\sigma}_{\exp }\left(x_{i} x_{j} s\right),
\end{array}
$$

in the notation of Ref. [3]. We will illustrate this formula with semi-analytical methods and the case of single $\mathrm{Z}$ production with the structure functions $\left\{F_{i}\right\}$ from Ref. [8] for definiteness. A MC realization will appear elsewhere [9].

As we have explained in Ref. [10], from the standpoint of using the result (1) with the structure functions $\left\{F_{i}\right\}$, we intend two possible approaches to the implied shower/ME matching [11]: one is based on $p_{T}$ matching and one based on the shower-subtracted residuals $\hat{\bar{\beta}}_{n, m}$. The respective shower can be that from PYTHIA [12], HERWIG [13], or the new shower algorithms in Ref. [14]. This combination of showers and exact ME's can be improved systematically to arbitrary precision order-by-order in $\left(\alpha_{s}, \alpha\right)$ in the presence of exact phase space [9].

To illustrate the size of the interplay between QED and QCD in the threshold region, we compute, with and without the QED, the respective ratio $r_{e x p}=\sigma_{e x p} / \sigma_{B o r n}$, with the results

$r_{\exp }= \begin{cases}1.1901 & , \mathrm{QCED} \equiv \mathrm{QCD}+\mathrm{QED}, \mathrm{LHC} \\ 1.1872 & , \mathrm{QCD}, \mathrm{LHC} \\ 1.1911 & , \mathrm{QCED} \equiv \mathrm{QCD}+\mathrm{QED}, \text { Tevatron } \\ 1.1879 & , \mathrm{QCD}, \text { Tevatron. }\end{cases}$

Here, we have used from (1) the $\tilde{\bar{\beta}}_{0,0}$-level result

$$
\begin{aligned}
& \hat{\sigma}_{\exp }\left(x_{1} x_{2} s\right)=\int_{0}^{v_{\max }} d v \gamma_{Q C E D} v^{\gamma_{Q C E D}-1} \\
& F_{Y F S}\left(\gamma_{Q C E D}\right) e^{\delta_{Y F S}} \hat{\sigma}_{B o r n}\left((1-v) x_{1} x_{2} s\right)(4)
\end{aligned}
$$

where we intend the well-known results for the respective parton-level Born cross sections and the value of $v_{\max }$ implied by the experimental cuts under study. The value for the $\mathrm{QED} \otimes \mathrm{QCD}$ exponent is $\gamma_{Q C E D}=\left\{2 Q_{f}^{2} \frac{\alpha}{\pi}+2 C_{F} \frac{\alpha_{s}}{\pi}\right\} L_{n l s}$ where $L_{n l s}=\ln x_{1} x_{2} s / \mu^{2}$ when $\mu$ is the factorization scale. The functions $F_{Y F S}\left(\gamma_{Q C E D}\right)$ and $\delta_{Y F S}\left(\gamma_{Q C E D}\right)$ are well-known [7] as well:

$$
F_{Y F S}\left(\gamma_{Q C E D}\right)=\frac{e^{-\gamma_{Q C E D} \gamma_{E}}}{\Gamma\left(1+\gamma_{Q C E D}\right)},
$$

with $\delta_{Y F S}\left(\gamma_{Q C E D}\right)=\frac{1}{4} \gamma_{Q C E D}+\left(Q_{f}^{2} \frac{\alpha}{\pi}+\right.$ $\left.C_{F} \frac{\alpha_{s}}{\pi}\right)\left(2 \zeta(2)-\frac{1}{2}\right)$, where $\zeta(2)$ is Riemann's zeta function of argument 2 , i.e., $\pi^{2} / 6$, and $\gamma_{E}$ is Euler's constant, i.e., $0.5772 \ldots$.

We see that the size of the QED effects, $\sim 0.3 \%$, is appreciable in a $1 \%$ precision tag budget and these effects are comparable to the QED effects found for the structure function evolution itself in Refs. [15-19]. We also see that our soft, QCD correction is entirely consistent with the exact results in Ref. [20-22] and that our QED effects are consistent with the exact $\mathcal{O}(\alpha)$ results in Refs. [23-25]. Such cross-checks are essential in establishing a rigorous precision tag on the theoretical predictions.

With an eye toward the $1 \%$ precision tag for the single heavy gauge boson production at the LHC, we have analyzed [26] the IR limit of the DGLAP [27] kernels themselves. The effects which we address are illustrated in Fig. 1. We apply the QCD exponentiation master formula from Ref. [4], the analog of (1) for just QCD, to the gluon emission transition that corresponds to $P_{q q}(z)$, i.e., to the squared amplitude for $q \rightarrow q(z)+G(1-z)$; as we show in Ref. [26], this allows us toget the following IR-improved DGLAP kernels

$$
\begin{aligned}
P_{q q}^{e x p}(z) & =C_{F} F_{Y F S}\left(\gamma_{q}\right) e^{\frac{1}{2} \delta_{q}}\left\{\frac{1+z^{2}}{1-z}(1-z)^{\gamma_{q}}\right. \\
& \left.-f_{q}\left(\gamma_{q}\right) \delta(1-z)\right\}
\end{aligned}
$$




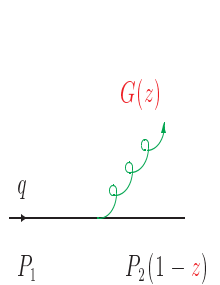

(a)

Figure 1. In (a), we show the usual process $q \rightarrow q(1-$ $z)+G(z)$; in (b), we show its multiple gluon improvement $q \rightarrow q(1-z)+G_{1}\left(\xi_{1}\right)+\cdots+G_{n}\left(\xi_{n}\right), \quad z=\sum_{j} \xi_{j}$.

$P_{G q}(z)^{e x p}=C_{F} F_{Y F S}\left(\gamma_{q}\right) e^{\frac{1}{2} \delta_{q}} \frac{1+(1-z)^{2}}{z} z^{\gamma_{q}}$,

$$
\begin{aligned}
P_{G G}^{e x p}(z) & =2 C_{G} F_{Y F S}\left(\gamma_{G}\right) e^{\frac{1}{2} \delta_{G}}\left\{\frac{1-z}{z} z^{\gamma_{G}}\right. \\
& +\frac{z}{1-z}(1-z)^{\gamma_{G}}+\frac{1}{2}\left(z^{1+\gamma_{G}}(1-z)\right. \\
& \left.\left.+z(1-z)^{1+\gamma_{G}}\right)-f_{G}\left(\gamma_{G}\right) \delta(1-z)\right\}
\end{aligned}
$$

$$
\begin{aligned}
P_{q G}^{e x p}(z) & =F_{Y F S}\left(\gamma_{G}\right) e^{\frac{1}{2} \delta_{G}} \frac{1}{2}\left\{z^{2}(1-z)^{\gamma_{G}}\right. \\
& \left.+(1-z)^{2} z^{\gamma_{G}}\right\}
\end{aligned}
$$

where from the standard DGLAP methodology [26] we have $f_{q}\left(\gamma_{q}\right)=\frac{2}{\gamma_{q}}-\frac{2}{\gamma_{q}+1}+\frac{1}{\gamma_{q}+2}$, $\gamma_{q}=C_{F} \frac{\alpha_{s}}{\pi} t=\frac{4 C_{F}}{\beta_{0}}, \delta_{q}=\frac{\gamma_{q}}{2}+\frac{\alpha_{s} C_{F}}{\pi}\left(\frac{\pi^{2}}{3}-\frac{1}{2}\right)$, $\gamma_{G}=C_{G} \frac{\alpha_{s}}{\pi} t=\frac{4 C_{G}}{\beta_{0}}, \delta_{G}=\frac{\gamma_{G}}{2}+\frac{\alpha_{s} C_{G}}{\pi}\left(\frac{\pi^{2}}{3}-\frac{1}{2}\right)$, and

$$
\begin{aligned}
f_{G}\left(\gamma_{G}\right) & =\frac{n_{f}}{C_{G}} \frac{1}{\left(1+\gamma_{G}\right)\left(2+\gamma_{G}\right)\left(3+\gamma_{G}\right)} \\
& +\frac{2}{\gamma_{G}\left(1+\gamma_{G}\right)\left(2+\gamma_{G}\right)} \\
& +\frac{1}{\left(1+\gamma_{G}\right)\left(2+\gamma_{G}\right)}+\frac{1}{2\left(3+\gamma_{G}\right)\left(4+\gamma_{G}\right)} \\
& +\frac{1}{\left(2+\gamma_{G}\right)\left(3+\gamma_{G}\right)\left(4+\gamma_{G}\right)} .
\end{aligned}
$$

Here, $\beta_{0}=11-\frac{2}{3} n_{f}$, where $n_{f}$ is the number of active quark flavors. We show in Ref $[26,28]$ that these improved kernels change significantly the evolution of the moments of the structure functions. For example, the non-singlet anomalous dimension
$A_{n}^{N S}$ becomes, from the exponentiated kernels,

$$
\begin{aligned}
& A_{n}^{N S}=C_{F} F_{Y F S}\left(\gamma_{q}\right) e^{\frac{1}{2} \delta_{q}} \\
& \quad\left[B\left(n, \gamma_{q}\right)+B\left(n+2, \gamma_{q}\right)-f_{q}\left(\gamma_{q}\right)\right]
\end{aligned}
$$

where $B(x, y)$ is the beta function given by $B(x, y)=\Gamma(x) \Gamma(y) / \Gamma(x+y)$. Compare the usual result

$$
A_{n}^{N S^{\circ}} \equiv C_{F}\left[-\frac{1}{2}+\frac{1}{n(n+1)}-2 \sum_{j=2}^{n} \frac{1}{j}\right] .
$$

The IR-improved n-th moment goes for large $n$ to a multiple of $-f_{q}$, consistent with $\lim _{n \rightarrow \infty} z^{n-1}=0$ for $0 \leq z<1$; the usual result diverges as $-2 C_{F} \ln n$. The two results differ for finite $n$ as well: we get, for example, for $\alpha_{s} \cong .118, A_{2}^{N S}=$ $C_{F}(-1.33), C_{F}(-0.966)$ for (12) and (11), respectively. See Refs. [26, 28] for further discussion.

Contact with the exact 2-loop and 3loop results in Refs. [29,30] has also been made [26]: for the non-singlet case, we have , in the notation of Ref. [30], the exact 3-loop IR-improved result

$$
\begin{array}{r}
P_{n s}^{+, e x p}(z)=\left(\frac{\alpha_{s}}{4 \pi}\right) 2 P_{q q}^{e x p}(z)+F_{Y F S}\left(\gamma_{q}\right) e^{\frac{1}{2} \delta_{q}} \\
{\left[\left(\frac{\alpha_{s}}{4 \pi}\right)^{2}\left\{(1-z)^{\gamma_{q}} \bar{P}_{n s}^{(1)+}(z)+\bar{B}_{2} \delta(1-z)\right\}\right.} \\
\left.+\left(\frac{\alpha_{s}}{4 \pi}\right)^{3}\left\{(1-z)^{\gamma_{q}} \bar{P}_{n s}^{(2)+}(z)+\bar{B}_{3} \delta(1-z)\right\}\right]
\end{array}
$$

where $P_{q q}^{\exp }(z)$ is given in (9) and the resummed residuals $\bar{P}_{n s}^{(i)+}, i=1,2$ are related to the exact results $[29,30]$ for $P_{n s}^{(i)+}, i=1,2$, via

$\bar{P}_{n s}^{(i)+}(z)=P_{n s}^{(i)+}(z)-B_{1+i} \delta(1-z)+\Delta_{n s}^{(i)+}(z)$,

where the $B_{i}[29,30]$ and the $\Delta_{n s}^{(i)+}(z)$ are given explicitly in Ref. [26]. The detailed phenomenological consequences of the fully exponentiated 2- and 3-loop DGLAP kernel set will appear elsewhere [9].

In summary, the methods illustrated and presented herein are all under investigation and implementation as we prepare for the era of $1 \%$ precision LHC theoretical predictions 
on the necessary processes. Finally, one of us ( B.F.L.W.) thanks Prof. W. Hollik for the support and kind hospitality of the MPI, Munich, while a part of this work was completed. We also thank Prof. S. Jadach for useful discussions. This work is partly supported by US DOE grant DE-FG02-05ER41399 and by NATO grant PST.CLG.980342.

\section{References}

1. M. Dittmar et al., Phys. Rev. D56, 7284 (1997); M. Rijssenbeek, in Proc. HCP2002, ed. M. Erdmann,(Karlsruhe, $2002)$ p. 424; M. Dittmar, ibid.,p. 431.

2. S. Klimenko, in Proc. HCP2002, ed. M. Erdmann,( Karlsruhe, 2002 ) p. 413.

3. C. Glosser et al.,Mod. Phys. Lett. A19, 2113 (2004); B.F.L. Ward et al., in Proc. DPF 2004, Int. J. Mod. Phys. A20, 3735 (2005); in Proc. ICHEP04, vol. 1, eds. H. Chen et al.,(World. Sci. Publ. Co., Singapore, 2005) p. 588.

4. B.F.L. Ward and S. Jadach, Acta Phys.Polon. B33, 1543 (2002); in Proc. ICHEP2002, ed. S. Bentvelsen et al.,( North Holland, Amsterdam, 2003 ) p. 275 ; Mod. Phys. Lett. A14, 491 (1999) ;D.B. DeLaney et al., Phys. Lett. B342, 239 (1995) ; Mod. Phys. Lett. A12, 2425 (1997); DPhys. Rev. D52, 108 (1995); ibid.D66, 019903(E) (2002).

5. J. Gatherall, Phys. Lett. B133(1983) 90.

6. D. R. Yennie et al., Ann. Phys. 13, 379 (1961); see also K. T. Mahanthappa, Phys. Rev. 126, 329 (1962).

7. See also S. Jadach et al., Comput. Phys. Commun. 102, 229 (1997); Phys. Rev. D55, 1206 (1997); Phys. Rev. D63, 113009 (2001); Comp. Phys. Commun. 130, 260 (2000); ibid.140, 432, 475 (2001).

8. A.D. Martin et al., Phys. Rev. D51, 4756 (1995).

9. S. Jadach et al., to appear.
10. B.F.L.

Ward and S.A. Yost, hep-ph/0509003, in Proc. HERA-LHC Workshop, CERN2005-014, DESY-PROC-2005-01, eds. A. De Roeck and H. Jung, (CERN, Geneva, 2005) p. 304.

11. S. Frixione and B. Webber, J. High Energy Phys. 0206, 029 (2002); S. Frixione et al., ibid.0308, 007 (2003).

12. T. Sjostrand et al., hep-ph/0308153.

13. G. Corcella et al., hep-ph/0210213.

14. S. Jadach and M. Skrzypek, Acta Phys. Pol. B35, 735 (2004); hep-ph/0504263, 0504205 .

15. S. Haywood, P.R. Hobson, W. Hollik and Z. Kunszt, in Proc. 1999 CERN Workshop on Standard Model Physics (and more) at the LHC, CERN-2000-004, eds. G. Altarelli and M.L. Mangano,( CERN, Geneva, 2000 ) p. 122.

16. H. Spiesberger, Phys. Rev. D52, 4936 (1995).

17. W.J. Stirling,"Electroweak Effects in Parton Distribution Functions", talk presented at ESF Exploratory Workshop, Electroweak Radiative Corrections to Hadronic Observables at TeV Energies, Durham, Sept., 2003.

18. M. Roth and S. Weinzierl, Phys. Lett. B590, 190 (2004).

19. W. J. Stirling et al., in Proc. ICHEP04, eds. H. Chen et al. (World Sci. Publ., Singapore, 2005) p. 527.

20. R. Hamberg et al., Nucl. Phys. B359, 343 (1991).

21. W.L. van Neerven and E.B. Zijlstra, Nucl. Phys. B382, 11 (1992); ibid. B680, 513 (2004).

22. C. Anastasiou et al.,Phys. Rev. D69, 094008 (2004).

23. U. Baur et al., Phys. Rev. D57, 199 (1998); ibid.D59, 013002 (1998); ibid.D65 (2002) 033007.

24. S. Dittmaier and M. Kramer, Phys. Rev. D65, 073007 (2002).

25. Z. Zykunov,Eur. Phys. J. C3, 9 (2001). 
26. B.F.L. Ward, hep-ph/0508140.

27. G. Altarelli and G. Parisi, Nucl. Phys. B126, 298 (1977); Yu. L. Dokshitzer, Sov. Phys. JETP 46, 641 (1977); L. N. Lipatov, Yad. Fiz. 20, 181 (1974); V. Gribov and L. Lipatov, Sov. J. Nucl. Phys. 15, 438, 675 (1972); see also J.C. Collins and J. Qiu, Phys. Rev. D39, 1398 (1989) for alternative discussion of the lowest order DGLAP theory.

28. B.F.L. Ward, hep-ph/0602025, in Proc. RADCOR05, Nucl. Phys. B Proc. Suppl. 157, 215 (2006).

29. E. G. Floratos et al., Nucl. Phys. B129, 66 (1977); ibid.B139, 545 (1978); ibid. B152, 493 (1979); A. Gonzalez-Arroyo et al., Nucl. Phys. B153, 161 (1979); A. Gonzalez-Arroyo and C. Lopez, Nucl. Phys. B166, 429 (1980); G. Curci et al., Nucl. Phys. B175, 27 (1980); W. Furmanski and R. Petronzio, Phys. Lett. B97, 437 (1980); E. G. Floratos et al., Nucl. Phys. B192, 417 (1981); R. Hamberg and W. Van Neerven, Nucl. Phys. B379, 143 (1992).

30. S. Moch et al., Nucl. Phys. B688, 101 (2004); ibid. B691, 129 (2004), and references therein. 